\title{
Effects of the Volume and Temperature on the Global and Segmental Dynamics in Poly(propylene glycol) and 1,4-Polyisoprene
}

\author{
C. M. ROLAND, ${ }^{1}$ M. PALUCH, ${ }^{3}$ R. CASAliNI ${ }^{1,2}$ \\ ${ }^{1}$ Chemistry Division, Naval Research Laboratory, Code 6120, Washington, DC 20375-5342 \\ ${ }^{2}$ Chemistry Department, George Mason University, Fairfax, Virginia 22030 \\ ${ }^{3}$ Institute of Physics, Silesian University, Uniwersytecka 4, 40-007 Katowice, Poland
}

Received 27 May 2004; revised 4 August 2004; accepted 31 August 2004

DOI: $10.1002 /$ polb.20287

Published online in Wiley InterScience (www.interscience.wiley.com).

\begin{abstract}
Published dielectric relaxation measurements for poly(propylene glycol) and 1,4-polyisoprene are analyzed to determine the relative effects that thermal energy and volume have on the temperature dependence of the normal-mode relaxation times, and these are compared with their effects on the temperature dependence of the local segmental relaxation times. For both polymers at temperatures well above the glasstransition temperature, both relaxation modes are governed more by the thermal energy than by the volume, although the latter's contribution is not negligible. Such a result is consistent with an assumption underlying models for polymer viscoelasticity, such as the Rouse and tube models, that the friction coefficient governing motions over large length scales can be identified with the local segmental friction coefficient. Moreover, the relaxation data for both the segmental and normal modes superimpose when expressed as a function of the product of the temperature and volume, the latter being raised to a power. This scaling form arises from an inverse power law for the repulsive part of the intermolecular potential. The value of the exponent on the volume is the same for the normal and segmental motions and for both polymers indicates a relatively soft potential. (C) 2004 Wiley Periodicals, Inc. J Polym Sci Part B: Polym Phys 42: 4313-4319, 2004
\end{abstract}

Keywords: density; dielectric relaxation; normal mode; polyisoprene; poly(propylene glycol); relaxation; segmental mode; rheology

\section{INTRODUCTION}

The rheology of polymeric materials has obvious practical significance and has been the focus of much research over the past half-century. The slow dynamics of entangled molecules is usually

Correspondence to: C. M. Roland (E-mail: roland@nrl. navy.mil)

Journal of Polymer Science: Part B: Polymer Physics, Vol. 42, 4313-4319 (2004) (C) 2004 Wiley Periodicals, Inc. interpreted in terms of reptation, ${ }^{1}$ which emphasizes the disparity in the spatial constraints on transverse motions, as opposed to motion along the chain backbone. The Doi-Edwards tube model $^{2}$ provides a theoretical framework for the reptation idea and, with various refinements over the past 25 years, has become predominant in the field. ${ }^{3,4}$ The tube model describes the entangled dynamics as Rouse chains moving in a network of topological constraints. There are two species-dependent parameters, the local (Rouse) friction co- 
efficient ( $s$ ) and a parameter characterizing the entanglements. For both the tube and Rouse models, the temperature dependence of the chain dynamics is contained in s. Because this is the same friction coefficient governing local segmental relaxation, ${ }^{5,6}$ ostensibly the implication is that the local modes and the chain modes should have the same temperature dependence. (This expectation is implicit in master curves for the dynamic properties of polymers that extend from the rubbery plateau into the glassy zone.) However, as shown by the breakdown of time-temperature superpositioning in the softening zone of the mechanical response, ${ }^{7-11}$ and by direct comparison of normal and segmental modes in the dielectric spectra of type-A polymers, ${ }^{12}$ the local dynamics and the chain modes do not always have the same temperature dependence. Within the scope of the tube and Rouse models, the discrepancy lies in the identification of $s$ with the friction coefficient for segmental relaxation.

Here we analyze published dielectric spectroscopy data to investigate local segmental relaxation (dielectric $\alpha$ process) and chain dynamics (dielectric normal-mode process) in poly(propylene glycol) (PPG) and polyisoprene (PI). Both polymers are barely entangled. PPG has a molecular weight of $4.0 \mathrm{~kg} / \mathrm{mol}$, and its entanglements are reputed to be abetted by transient coupling via hydrogen bonding of the chain ends. ${ }^{13}$ For PI, the weight-average molecular weight is $11.1 \mathrm{~kg} /$ mol, which is larger than the entanglement molecular weight by almost a factor of two. ${ }^{8} \mathrm{PPG}$ and PI are both type-A polymers, having a permanent dipole moment parallel to the chain, which means that global motions of the backbone are dielectrically active. ${ }^{14,15}$ Many dielectric studies of $\mathrm{PPG}^{16-21}$ and $\mathrm{PI}^{8,12,22-25}$ have been carried out at ambient pressure, but only a few have been performed at elevated pressures. ${ }^{26-29}$ Recently, dielectric relaxation times for the normal mode $\left(\tau_{\mathrm{N}}\right)$ and for local segmental relaxation $\left(\tau_{\alpha}\right)$ were measured for $\mathrm{PPG}^{30}$ and $\mathrm{PI}^{31}$ as a function of temperature and pressure. Although the focus of the tube and Rouse models is the rheological response, dielectric spectroscopy probes the chain dynamics in an analogous fashion as mechanical measurements. ${ }^{8,32}$ Thus, although the shape of the respective relaxation spectra can $\operatorname{differ}^{4}$, the temperature and pressure dependences of the relaxation times as measured by the two methods are the same..$^{8,10,31,33,34}$ By combining the dielectric results for these two materials with equationof-state (pressure-volume-temperature) data, we can obtain the volume dependence of the relaxation times. More specifically, we can assess the effects of changes in thermal energy and volume on the respective temperature dependences of $\tau_{\alpha}$ and $\tau_{\mathrm{N}}$.

Previously, this type of analysis has been carried out only for the segmental relaxation process. ${ }^{35-44}$ For small-molecule van der Waals liquids, the contributions to $\tau_{\alpha}(T)$ from temperature and volume are comparable, as reflected in the magnitudes of the isobaric and isochoric activation energies. ${ }^{43,44}$ On the other hand, for polymers, temperature generally exerts a more dominant influence on the segmental relaxation. However, the effect of volume is not negligible and becomes more important for more flexible chain polymers, such as siloxanes. ${ }^{36}$ For strongly hydrogen-bonded materials, temperature tends to become overwhelmingly the dominant parameter controlling the temperature dependence of the relaxation times.

In this work, we extend the analysis of temperature and volume effects to consider the normal mode. We also employ a recently proposed scaling of the relaxation times that is based on an inverse power form for the intermolecular repulsive potential. ${ }^{45}$ We find that for PPG and PI well above the glass-transition temperature $\left(T_{\mathrm{g}}\right), \tau_{\alpha}$ and $\tau_{\mathrm{N}}$ are governed similarly by temperature and volume. Accordingly, master curves for each have been obtained, with the same scaling exponent for the two relaxation times. Our findings give strong support to a basic tenet of the tube and Rouse models, that is, the use of the local segmental friction factor in the description of the global (chain) dynamics.

\section{RESULTS}

\section{PPG}

We have previously reported the segmental-mode and normal-mode relaxation times for PPG, measured from ambient pressure to pressures as high as $1.2 \mathrm{GPa}$, at five temperatures (from 258 to 313 $\mathrm{K}) .{ }^{30}$ The relaxation times are defined as the inverse of the angular frequency associated with the maximum in the dielectric loss and are close to the average relaxation times, if the peaks are not broad. For this molecular weight of PPG (4 $\mathrm{kg} / \mathrm{mol}$ ), there is a substantial difference between $\tau_{\alpha}$ and $\tau_{\mathrm{N}}$. However, because the segmental relaxation times are more sensitive to both tempera- 


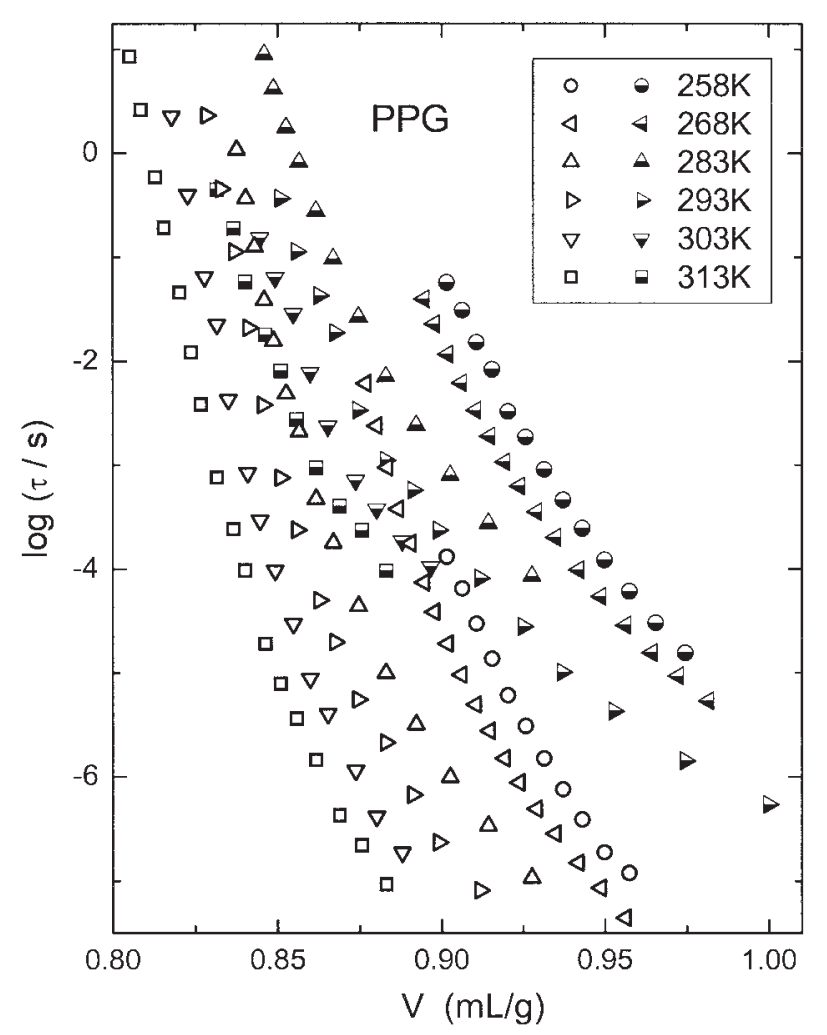

Figure 1. Segmental-mode relaxation times (hollow symbols) and normal-mode relaxation times (half-filled symbols) for PPG as a function of the specific volume $(V)$. The measurements were carried out at the indicated temperatures at various pressures ${ }^{30,46}$ and at ambient pressure as a function of temperature. ${ }^{25}$

ture $^{19}$ and pressure ${ }^{29,30}$ than the normal-mode relaxation times are, conditions can be found under which the two relaxation processes can be measured simultaneously. We convert the isotherms [isothermal dielectric relaxation times as a function of pressure] from ref. 30 into a function of volume by using the equation state for PPG: ${ }^{30}$

$$
\begin{gathered}
V(T, P)=\left(0.9852+7.2 \times 10^{-4} T+4.710^{-7} T^{2}\right) \\
\times\left(1-0.0894 \ln \left(1+\frac{P}{171 \exp (-0.0052 T)}\right)\right)
\end{gathered}
$$

where $V$ is the specific volume $(\mathrm{mL} / \mathrm{g}), P$ is the pressure $(\mathrm{MPa})$, and $T$ is the temperature $\left({ }^{\circ} \mathrm{C}\right)$. The results are plotted in Figure $1 .^{46}$ It can be seen that relaxation times for either process are not uniquely defined by the volume; thermal energy obviously exerts an influence. However, $\tau_{\alpha}$ and $\tau_{\mathrm{N}}$ are also changing with pressure for any fixed temperature, and this means that volume also plays a role.

We can quantify the relative effects of temperature and volume from the ratio of the thermal expansivity measured at a constant value of the relaxation time, $\alpha_{\tau}=\left.V^{-1} \frac{\partial V}{\partial T}\right|_{\tau}$, to the isobaric value of the thermal expansion coefficient, $\alpha_{p}$ $=\left.V^{-1} \frac{\partial V}{\partial T}\right|_{p} \cdot{ }^{42}$ This ratio, $\mid \alpha_{\tau} / \alpha_{\mathrm{P}}$ (because $\alpha_{\tau}$ is less than 0 , the absolute value is the quantity of interest), measures how much the volume has to change (by a variation of pressure) for the relaxation time to remain constant when the temperature is varied. Thus, $\left|\alpha_{\tau}\right| \alpha_{\mathrm{P}}$ is 1 if temperature (thermal energy) and volume exert equivalent effects on the temperature dependence of the relaxation times, whereas the ratio is much larger than one if temperature is the more dominant control variable. ${ }^{42}$ The expansivity ratio is related to the apparent activation enthalpies (slopes of the Arrhenius curves) at constant volume $\left(H_{\mathrm{V}}\right)$ and constant pressure $\left(E_{\mathrm{P}}\right)$ as $\left|\alpha_{\tau}\right| / \alpha_{\mathrm{P}}=-H_{\mathrm{V}} /\left(E_{\mathrm{P}}\right.$ $\left.-H_{\mathrm{V}}\right){ }^{39}$ The quantity $H_{\mathrm{V}} / E_{\mathrm{P}}$ is another measure of the relative effect of temperature and volume on $\tau(T){ }^{41}$

For PPG at ambient pressure near $T_{\mathrm{g}}(\sim 202$ $\mathrm{K}),\left|\alpha_{\tau}\right| \alpha_{\mathrm{P}}$ is $2.0 \pm 0.4,{ }^{30}$ and this means that for low temperatures, thermal energy exerts roughly twice the influence that the specific volume does in determining the change in $\tau_{\alpha}$ with temperature. This result is consistent with the early work of Williams. ${ }^{47}$ Near $T_{\mathrm{g}}$, the chain modes are too slow to be measured dielectrically. To compare the temperature and volume effects on both $\tau_{\alpha}$ and $\tau_{\mathrm{N}}$ at the same temperature, we calculate the expansivity ratios for the two processes at a higher temperature $(288 \mathrm{~K})$ and an elevated pressure (186 MPa). These particular conditions avoid the need of extrapolating any of the data in Figure 1. From eq $1, \alpha_{\mathrm{P}}=4.72 \times 10^{-4} \mathrm{~K}^{-1}$. The isochronal thermal expansion coefficient is calculated at $288 \mathrm{~K}$ and pressures at which $\tau_{\mathrm{N}}$ is fixed at $0.01 \mathrm{~s}$; this yields $\alpha_{\tau, \mathrm{N}}=-1.30( \pm 0.1) \times 10^{-3}$ $\mathrm{K}^{-1}$. At $288 \mathrm{~K}$ and $186 \mathrm{MPa}$, the segmental relaxation time is $2.0 \times 10^{-5} \mathrm{~s}$. The volume expansivity at which $\tau_{\alpha}$ is fixed at this value is calculated to be $\alpha_{\tau, \alpha}=-1.25( \pm 0.2) \times 10^{-3} \mathrm{~K}^{-1}$. As summarized in Table 1 , the ratio of these quantities is then obtained as $\left|\alpha_{\tau, \mathrm{N}}\right| / \alpha_{\mathrm{P}}=2.8 \pm 0.2$ for the normal mode and as $\left|\alpha_{\tau, \alpha}\right| / \alpha_{\mathrm{P}}=2.6 \pm 0.3$ for the segmental mode. 
Table 1. Results for PPG and PI

\begin{tabular}{|c|c|c|c|c|c|c|c|}
\hline & $T$ & $P$ & $\tau_{\mathrm{N}}$ & $\tau_{\alpha}$ & $-\alpha_{\tau, \mathrm{N}} / \alpha_{\mathrm{P}}$ & $-\alpha_{\tau, \alpha} / \alpha_{\mathrm{P}}$ & $\gamma$ \\
\hline PPG & $258 \mathrm{~K}$ & $186 \mathrm{MPa}$ & $0.01 \mathrm{~s}$ & $2.0 \times 10^{-5} \mathrm{~s}$ & $2.8 \pm 0.2$ & $2.6 \pm 0.3$ & $2.5 \pm 0.35$ \\
\hline PI & $283 \mathrm{~K}$ & $200 \mathrm{MPa}$ & $0.1 \mathrm{~s}$ & $3.1 \times 10^{-6} \mathrm{~s}$ & $3.0 \pm 0.3$ & $3.3 \pm 0.4$ & $3.0 \pm 0.25$ \\
\hline
\end{tabular}

The expansivity ratio for the segmental mode, $\left|\alpha_{\tau, \alpha}\right| \alpha_{\mathrm{P}}=2.6$, is larger than the value at $T_{\mathrm{g}},{ }^{30}$ indicating that the relative contribution from thermal energy increases at higher temperatures. In fact, we find there is a consistent trend of slightly increasing $\left|\alpha_{\tau, \alpha}\right| / \alpha_{\mathrm{P}}$ with decreasing $\tau_{\alpha}$. Of greater interest here is the finding that within the experimental error, local segmental relaxation and the normal mode are governed equivalently by temperature and volume: $\left|\alpha_{\tau, \alpha}\right| / \alpha_{\mathrm{P}} \approx\left|\alpha_{\tau, \mathrm{N}}\right| / \alpha_{\mathrm{P}}$ $\approx 2.7$. That is, roughly three-quarters of the increase in either relaxation time with increasing temperature is a direct result of greater thermal energy, the remaining increase in $\tau$ due to the accompanying volume expansion. It is interesting that even though both the temperature and pressure dependences of the two relaxation times differ, at least in the regime in which $\tau_{\mathrm{N}}$ is approximately $0.01 \mathrm{~s}$ and $\tau_{\alpha}$ is about 3 decades shorter, the relative effects of thermal energy and volume are the same for the two processes. The larger variation in $\tau_{\alpha}$ with a change in either temperature or volume is due to the greater sensitivity of the local segmental dynamics to these variables, not to a difference in their relative effect on $\tau_{\alpha}$. The relatively weak effect of the volume on $\tau_{\alpha}(T)$ and $\tau_{\mathrm{N}}(T)$ is ostensibly at odds with free-volume interpretations of polymer dynamics; however, the free volume and the specific volume are not equivalent. In fact, the former can even change at fixed volume. $^{5}$

\section{PI}

Floudas and Reisinger ${ }^{31}$ reported isotherms for both $\tau_{\alpha}$ and $\tau_{\mathrm{N}}$ of PI measured at a series of temperatures at ambient pressure and at two temperatures for pressures up to $350 \mathrm{MPa}$. The reported Vogel-Fulcher fits to these data are plotted in Figure 2 as a function of the specific volume, the latter determined from the equation of state for PI: ${ }^{31}$

$$
\begin{aligned}
V(T, P)=( & 1.0943+6.293 \times 10^{-4} T+6.231 \\
& \left.\times 10^{-7} T^{2}\right)(1-0.0894 \mathrm{ln} \\
& \left.\times\left(1+\frac{P}{202 \exp (-0.004653 T)}\right)\right)
\end{aligned}
$$

Figure 2 shows that temperature and volume both affect the temperature dependence of the relaxation times. Just as for PPG, we calculate the isobaric and isochronal thermal expansion coefficients. To avoid any extrapolation, we choose

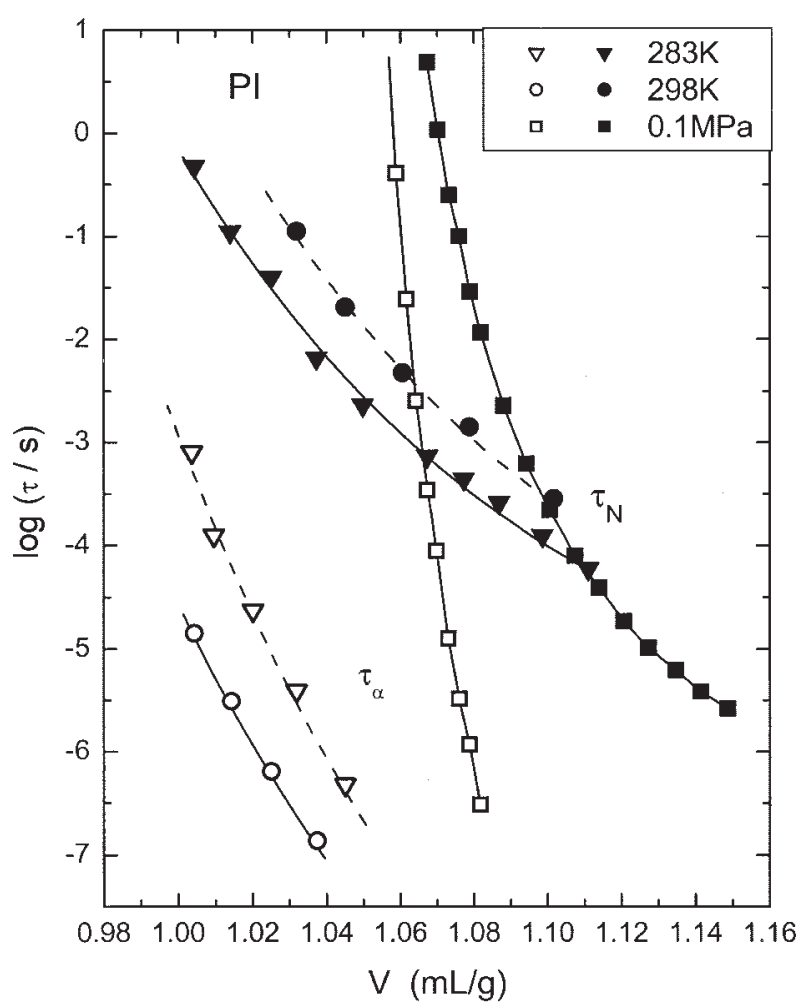

Figure 2. Segmental-mode relaxation times (hollow symbols) and normal-mode relaxation times (filled symbols) for PI as a function of the specific volume $(V)$. The measurements ${ }^{31}$ were carried out isobarically at atmospheric pressure and isothermally at the two indicated temperatures at pressures of 0.1-350 MPa for $\tau_{\mathrm{N}}$ and 150-350 MPa for $\tau_{\alpha}$. 
$263 \mathrm{~K}$ and $200 \mathrm{MPa}$, for which $\alpha_{\mathrm{P}}$ is $3.589 \times 10^{-4}$ $\mathrm{K}^{-1}$. The relaxation times at this temperature and pressure are $\tau_{\mathrm{N}}=0.01 \mathrm{~s}$ and $\tau_{\alpha}=3.1 \times 10^{-6}$ $\mathrm{s}$, for which the corresponding isochronal expansivities are calculated to be $\alpha_{\tau, \mathrm{N}}=-1.08( \pm 0.1)$ $\times 10^{-3} \mathrm{~K}^{-1}$ and $\alpha_{\tau, \alpha}=-1.18( \pm 0.15) \times 10^{-3} \mathrm{~K}^{-1}$. From these data, we calculate the expansivity ratios listed in Table 1; within the experimental error, we find $\left|\alpha_{\tau, \mathrm{N}}\right| / \alpha_{\mathrm{P}}=\left|\alpha_{\tau, \alpha}\right| / \alpha_{\mathrm{P}} \approx 3.2$. Thus, similar to the case of PPG, the effects of temperature and volume are the same for the two relaxation processes. Also, at this relatively high temperature, thermal energy has a stronger influence on the relaxation times than the volume does. Floudas and Reisinger ${ }^{31}$ reached a similar conclusion specifically for the $\alpha$ relaxation at lower temperatures, near $T_{\mathrm{g}}$.

\section{Scaling of the Relaxation Times}

There is no accurate theoretical prediction for the combined temperature and pressure dependences of either the segmental-mode or normal-mode relaxation times. Although $\tau_{\alpha}(T)$ data are known to conform to the Vogel-Fulcher (or Williams-Landel-Ferry) equation up to about $1.3 \times T_{\mathrm{g}}$, the origin of this behavior is uncertain, with different models, based variously on free volume $e^{48-50}$ or entropy, ${ }^{51-53}$ showing limited success. For the pressure dependence of $\tau_{\alpha}$, the situation is worse, with different models yielding disparate forms for $\tau_{\alpha}(P)$. Concerning the chain dynamics, there is only the inference from the Rouse and tube models that $\tau_{\mathrm{N}}$ should follow the temperature and pressure dependences of segmental relaxation.

We recently proposed a generalized scaling of $\tau_{\alpha}$ data obtained for both temperature and pressure. It is based on an inverse power law, $\phi(r) \propto$ $r^{-3 \gamma}$, for the repulsive potential, where $r$ is the intermolecular separation and $\gamma$ is a material parameter. ${ }^{54}$ The underlying idea is that the liquid structure is primarily determined by repulsive forces, with the attractive forces serving as a mean background potential that holds the molecules together ${ }^{55,56}$ With this form, in principle all thermodynamic properties of the material can be expressed as a function of the variable $T^{-1} V^{-\gamma} .57$ In practice, a power potential is of more limited validity. For the equation of state, for example, it will break down because of neglect of the attractive part of the intermolecular interactions. We have found that dynamic quantities related to the glass-transition relaxation, such as $\tau_{\alpha}$, the ionic conductivity, and the viscosity of monomeric glass formers, can be expressed as a single function of the variable $T^{-1} V^{-\gamma}{ }^{45}$ Moreover, the magnitude of $\gamma$ is correlated with the relative contribution of temperature and volume to the local dynamics. For the extreme cases, hard spheres (volumedominated) and thermally activated dynamics, $\gamma$ $=\infty$ and 0 , respectively. For various glass formers, including van der Waals molecules, associated liquids, and polymers, we find $0.1<\gamma<9$, which parallels the magnitude of $\left|\alpha_{\tau}\right| / \alpha_{\mathrm{P}}$. An exponent as small as 0.1 , as found for strongly associated liquids, is unrealistic, reflecting the inadequacy of a repulsive power potential for fluids characterized by extensive hydrogen bonding.

If this scaling applies to the global dynamics, which has not heretofore been shown, our results suggest that (1) for each polymer, $\tau_{\alpha}$ and $\tau_{\mathrm{N}}$ should exhibit the same scaling because we find $\left|\alpha_{\tau, \alpha}\right| / \alpha_{\mathrm{P}} \approx\left|\alpha_{\tau, \mathrm{N}}\right| / \alpha_{\mathrm{P}}$ and (2) because the relative effects of the temperature are roughly comparable for PI and PPG, their respective scaling exponents should be similar.

In Figure $3, \tau_{\alpha}$ and $\tau_{\mathrm{N}}$ for the two polymers are displayed as a function of $T^{-1} V^{-\gamma}$. For both modes, the same value of $\gamma$ causes the relaxation times for various temperatures and pressures to fall on a single curve. Over as much as 8 decades, the superpositioning is quite good. We also find that the scaling exponents, $\gamma=2.5 \pm 0.35$ for PPG and $\gamma=3.0 \pm 0.15$ for PI, are close, in agreement with the approximate equivalence of their respective $\left|\alpha_{\tau}\right| / \alpha_{\mathrm{P}}$ values. These values of $\gamma$ are fairly small, reflecting the softness of the intermolecular potential for these polymers.

\section{CONCLUSIONS}

For two barely entangled polymers, at temperatures for which the normal-mode relaxation time is $0.01-0.1 \mathrm{~s}$, the respective temperature dependences of the segmental and normal modes are governed very similarly by thermal energy and volume. This result is congruent with an implicit assumption of the Rouse and tube models, that the relevant friction coefficient for the global dynamics can be identified with the friction coefficient for local segmental relaxation. However, our results ostensibly contradict the fact that the segmental and chain modes have different temperature $^{7-10}$ and pressure dependences. ${ }^{30,31}$ However, the same relative influence from thermal energy and volume on $\tau_{\alpha}$ and $\tau_{\mathrm{N}}$ does not require the magnitude of the change induced in either relax- 


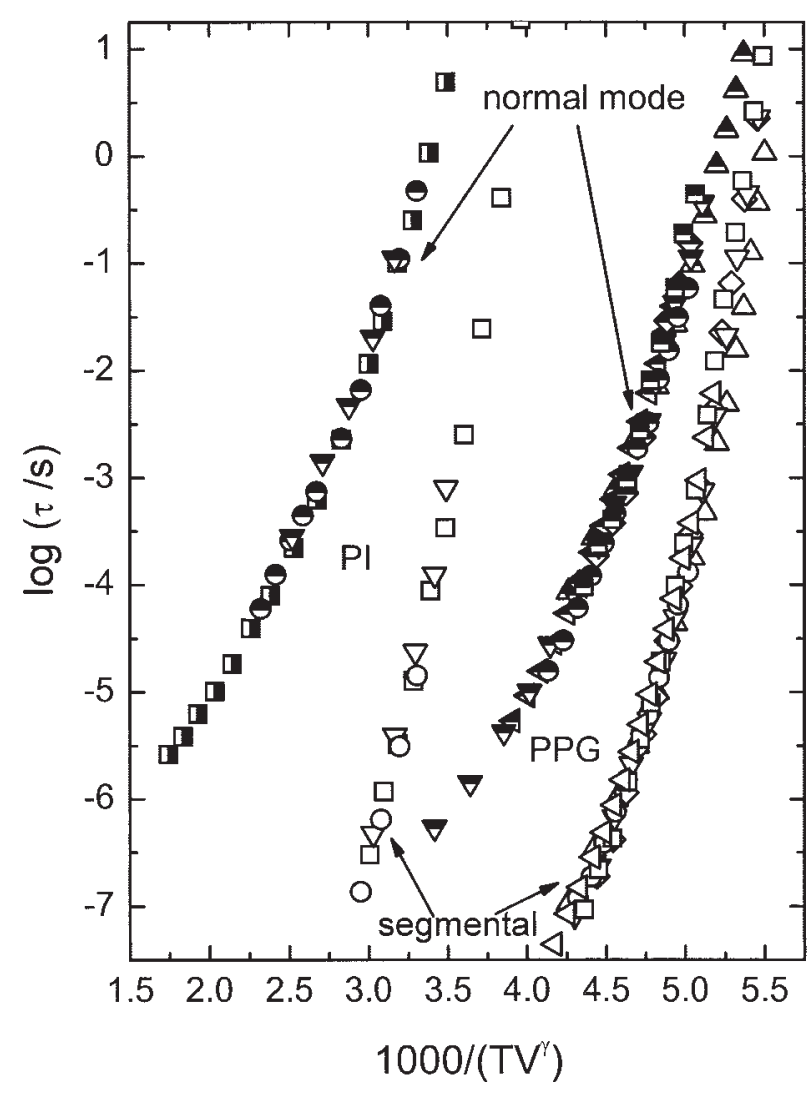

Figure 3. Relaxation times from Figures 1 and 2 as a function of $T^{-1} V^{-\gamma}$, where $\gamma=2.5$ and 3.0 for PPG and PI, respectively. For PPG, the temperature was $(\bigcirc)$ 258, $(\triangleleft) 268,(\triangle) 283,(\nabla) 293,(\diamond) 303$, or $(\square) 313 \mathrm{~K}$. For $\mathrm{PI}$, the temperature was $(\nabla) 283$ or $(\bigcirc) 298 \mathrm{~K}$, and the pressure was ( $\square$ ) $0.1 \mathrm{MPa}$.

ation time to be the same. For segmental relaxation, the effects may be amplified, for example, by intermolecular interactions, in the manner described by the coupling model. ${ }^{11,58}$

Similar to the literature results for segmental relaxation near $T_{\mathrm{g}}$ in other polymers, we find for both PPG and PI that thermal energy exerts a more significant effect on the temperature dependence of $\tau_{\alpha}$ than the volume does. This implies a relatively soft intermolecular potential, and indeed, superpositioning of the relaxation times is achieved with a small value of the scaling exponent. This scaling exponent comes from an inverse power form for the intermolecular potential.

Finally, we note that our analysis was carried out for temperatures well above $T_{\mathrm{g}}$. At these high temperatures, the segmental relaxation times are small $\left(\tau_{\alpha}<1 \mathrm{~s}\right)$. When $\tau_{\alpha}$ becomes short, the temperature dependences of the segmental and chain modes are expected to be comparable. ${ }^{10}$
However, as seen in these data, differences in pressure dependences are still observed. It would certainly be interesting to extend the experimental work to lower temperatures.

This work was supported by the Office of Naval Research. The authors thank G. M. Poliskie and K. L. Ngai for useful comments.

\section{REFERENCES AND NOTES}

1. de Gennes, P. G. J Chem Phys 1971, 55, 572-579.

2. Doi, M.; Edwards, S. F. The Theory of Polymer Dynamics; Clarendon: Oxford, 1986.

3. Lodge, T. P.; Rotstein, N. A.; Prager, S. Adv Chem Phys 1990, 79, 1-132.

4. Watanabe, H. Prog Polym Sci 1999, 24, 1253-1403.

5. Ferry, J. D. Viscoelastic Properties of Polymers; Wiley: New York, 1980.

6. Yamakawa, H. Modern Theory of Polymer Solutions; Harper \& Row: New York, 1971.

7. Plazek, D. J. J Phys Chem 1965, 69, 3480.

8. Santangelo, P. G.; Roland, C. M. Macromolecules 1998, 31, 3715-3719.

9. Plazek, D. J.; Chay, I. C.; Ngai, K. L.; Roland, C. M. Macromolecules 1995, 28, 6432-6436.

10. Roland, C. M.; Ngai, K. L.; Santangelo, P. G.; Qiu, X. H.; Ediger, M. D.; Plazek, D. J. Macromolecules 2001, 34, 6159-6160.

11. Ngai, K. L.; Plazek, D. J. Rubber Chem Technol 1995, 68, 376-434.

12. Schonhals, A. Macromolecules 1993, 26, 1309.

13. Fleischer, G.; Helmstedt, M.; Alig, I. Polym Commun 1990, 31, 409-411.

14. Stockmayer, W. H. Pure Appl Chem 1967, 15, 539.

15. Adachi, K.; Kotaka, T. Prog Polym Sci 1993, 18, 585-622.

16. Baur, M. E.; Stockmayer, W. H. J Chem Phys 1965, 43, 4319.

17. Schonhals, A.; Stauga, R. J Chem Phys 1998, 108, 5130-5136.

18. Leon, C.; Ngai, K. L.; Roland, C. M. J Chem Phys 1999, 110, 11585-11591.

19. Ngai, K. L.; Schonhals, A.; Schlosser, E. Macromolecules 1992, 25, 4915-4919.

20. Schonhals, A.; Kremer, F. J Non-Cryst Solids 1994, 172, 336-343.

21. Johari, G. P. Polymer 1986, 27, 866-870.

22. Adachi, K.; Kotaka, T. Macromolecules 1984, 17, 120-122.

23. Roland, C. M.; Bero, C. A. Macromolecules 1996, 29, 7521-7526.

24. Adachi, K.; Kotaka, T. Macromolecules 1985, 18, 466-472.

25. Boese, D.; Kremer, F. Macromolecules 1990, 23, 829-835. 
26. Dalal, E. N.; Phillips, P. J. Macromolecules 1983, 16, 890-897.

27. Suzuki, A.; Masuko, M.; Wakisaka, K. Tribol Int 2002, 35, 55-63.

28. Casalini, R.; Roland, C. M. J Chem Phys 2003, 119, 11951-11956.

29. Andersson, S. P.; Andersson, O. Macromolecules 1998, 31, 2999-3006.

30. Roland, C. M.; Psurek, T.; Pawlus, S.; Paluch, M. J Polym Sci Part B: Polym Phys 2003, 41, 30473052.

31. Floudas, G.; Reisinger, T. J Chem Phys 1999, 111, 5201-5204.

32. Watanabe, H.; Yao, M. L.; Osaki, K. Macromolecules 1996, 29, 970103.

33. McCrum, N. G.; Read, B. E.; Williams, G. Anelastic and Dielectric Effects in Polymeric Solids; Dover: New York, 1991.

34. Floudas, G.; Gravalides, C.; Reisinger, T.; Wegner, G. J Chem Phys 1999, 111, 9847-9852.

35. O’Reilly, J. M. J Polym Sci 1970, 57, 429.

36. Paluch, M.; Casalini, R.; Patkowski, A.; Pakula, T.; Roland, C. M. Phys Rev E 2003, 68, 031802.

37. Paluch, M.; Roland, C. M.; Casalini, R.; Meier, G.; Patkowski, A. J Chem Phys 2003, 118, 4578-4582.

38. Roland, C. M.; Capaccioli, S.; Lucchesi, M.; Casalini, R. J Chem Phys 2004, 120, 10640-10646.

39. Casalini, R.; Roland, C. M. J Chem Phys 2003, 119, 4052-4059.

40. Roland, C. M.; Casalini, R.; Santangelo, P.; Sekula, M.; Ziolo, J.; Paluch, M. Macromolecules 2003, 36, 4954-4959.

41. Williams, G. In Dielectric Spectroscopy of Polymeric Materials; Runt, J. P.; Fitzgerald, J. J., Eds.; American Chemical Society: Washington, DC, 1997.
42. Ferrer, M. L.; Lawrence, C.; Demirjian, B. G.; Kivelson, D.; Alba-Simionesco, C.; Tarjus, G. J Chem Phys 1998, 109, 8010-8015.

43. Paluch, M.; Casalini, R.; Roland, C. M. Phys Rev B 2002, 66, 092202.

44. Roland, C. M.; Paluch, M.; Pakula, T.; Casalini, R. Philos Mag B 2004, 84, 1573.

45. Casalini, R.; Roland, C. M. Phys Rev E 2004, 69, 062501.

46. The data for PPG at $283 \mathrm{~K}$ in ref. 30 had significant scatter; thus, they were remeasured.

47. Williams, G. Trans Faraday Soc 1965, 61, 1564.

48. Cohen, M. H.; Grest, G. S. Phys Rev B 1979, 20, 1077-1098.

49. Paluch, M.; Casalini, R.; Roland, C. M. Phys Rev E 2003, 67, 021508.

50. Corezzi, S.; Capaccioli, S.; Casalini, R.; Fioretto, D.; Paluch, M.; Rolla, P. A. Chem Phys Lett 2000, 320, 113-117.

51. Adam, G.; Gibbs, J. H. J Chem Phys 1965, 43, 139.

52. Casalini, R.; Paluch, M.; Fontanella, J. J.; Roland, C. M. J Chem Phys 2002, 117, 4901-4906.

53. Casalini, R.; Capaccioli, S.; Lucchesi, M.; Rolla, P. A.; Corezzi, S. Phys Rev E 2001, 63, 031207.

54. Hoover, W. G.; Ross, M. Contemporary Phys 1971, 12, 339-356.

55. Weeks, J. D.; Chandler, D.; Andersen, H. C. J Chem Phys 1971, 54, 5237-5247.

56. Shell, M. S.; Debenedetti, P. G.; La Nave, E.; Sciortino, F. J Chem Phys 2003, 118, 8821-8830.

57. March, N. H.; Tosi, M. P. Introduction to Liquid State Physics; World Scientific: Singapore, 2002.

58. Ngai, K. L.; Roland, C. M. Macromolecules 1993, 26, 6824-6830. 